\title{
CHERN CLASS FORMULAS FOR QUIVER VARIETIES
}

\author{
ANDERS SKOVSTED BUCH AND WILLIAM FULTON
}

\section{INTRODUCTION}

Our goal in this paper is to prove a formula for the general degeneracy locus $\Omega_{r}$ associated to an oriented quiver of type $A_{n}$. If we are given a sequence of vector bundles and vector bundle maps

$$
E_{0} \stackrel{\phi_{1}}{\longrightarrow} E_{1} \stackrel{\phi_{2}}{\longrightarrow} E_{2} \rightarrow \cdots \rightarrow E_{n-1} \stackrel{\phi_{n}}{\longrightarrow} E_{n}
$$

on an algebraic variety $X$, and a collection $r=\left(r_{i j}\right)_{0 \leq i<j \leq n}$ of non-negative integers, there is a degeneracy locus $\Omega_{r}=\Omega_{r}\left(E_{\bullet}\right)=\Omega_{r}\left(E_{\bullet}, \phi_{\bullet}\right)$ defined by

$$
\Omega_{r}=\left\{x \in X \mid \operatorname{rank}\left(E_{i}(x) \rightarrow E_{j}(x)\right) \leq r_{i j} \forall i<j\right\} .
$$

This is a closed subscheme of $X$; locally, where the bundles are trivial, this is defined by vanishing of the minors of size $r_{i j}+1$ in the product of matrices giving the map $\phi_{j} \circ \cdots \circ \phi_{i+1}$ from $E_{i}$ to $E_{j}$, for all $i<j$.

Not all rank conditions give reasonable loci. Those that do - and the only ones we will consider - are characterized by the conditions

$$
\begin{gathered}
r_{i j} \leq r_{i, j-1} \quad \text { and } \quad r_{i j} \leq r_{i+1, j} \quad \text { for all } i<j, \text { and } \\
r_{i+1, j-1}-r_{i, j-1}-r_{i+1, j}+r_{i j} \geq 0 \quad \text { for all } i<j-1,
\end{gathered}
$$

where we set $r_{i i}=\operatorname{rank}\left(E_{i}\right)$. In fact, rank conditions satisfying (1.2) are the only conditions that can actually occur, i.e. for which one can have equality in (1.1). When the maps are sufficiently generic, each such $\Omega_{r}$ is irreducible, of codimension

$$
d(r)=\sum_{i<j}\left(r_{i, j-1}-r_{i j}\right)\left(r_{i+1, j}-r_{i j}\right) .
$$

When $n=1$, the formula for $\Omega_{r}$ is the well-known Giambelli-Thom-Porteous formula, which we recall in order to introduce some notation. For a map $\phi: E \rightarrow F$ of vector bundles of ranks $e$ and $f$, and a non-negative integer $r \leq \min (e, f), \Omega_{r}$ is the locus where $\phi$ has rank at most $r$. The formula for $\Omega_{r}$ is the Schur polynomial

$$
s_{(e-r)^{f-r}}(F-E),
$$

which is defined as follows. Define cohomology classes $h_{i}$ by the formula $\sum h_{i}=$ $c\left(E^{\vee}\right) / c\left(F^{\vee}\right)$, where $c\left(E^{\vee}\right)=1-c_{1}(E)+c_{2}(E)-\cdots$ is the total Chern class, and the division is carried out formally; in particular, $h_{0}=1$ and $h_{i}=0$ for $i<0$. For any sequence $\lambda=\left(\lambda_{1}, \ldots, \lambda_{p}\right)$ of non-negative integers, set

$$
s_{\lambda}(F-E)=\operatorname{det}\left(h_{\lambda_{i}+j-i}\right)_{1 \leq i, j \leq p} .
$$

In the Giambelli-Thom-Porteous formula, $\lambda=(e-r)^{f-r}$ denotes the sequence $e-r$ repeated $f-r$ times. In a Schur determinant $s_{\lambda}(F-E), \lambda$ will usually be a

Date: November 9, 2018.

The research of the second author was supported by an Erlander Professorship in Sweden and the National Science Foundation. 
partition, i.e. a weakly decreasing sequence, but later we will also need this notation when $\lambda$ is not a partition.

Our general formula for the locus $\Omega_{r}$, when $r$ is any set of rank conditions satisfying (1.2), has the form

$$
\sum_{\lambda} c_{\lambda}(r) s_{\lambda}\left(E_{\bullet}\right)
$$

where the sum is over sequences $\lambda=(\lambda(1), \lambda(2), \ldots, \lambda(n))$, with each $\lambda(i)$ a partition. The class $s_{\lambda}\left(E_{\bullet}\right)$ is defined to be

$$
s_{\lambda}\left(E_{\bullet}\right)=s_{\lambda(1)}\left(E_{1}-E_{0}\right) \cdot s_{\lambda(2)}\left(E_{2}-E_{1}\right) \cdot \ldots \cdot s_{\lambda(n)}\left(E_{n}-E_{n-1}\right) .
$$

The coefficients $c_{\lambda}(r)$ are certain integers for which we give an inductive formula.

A second purpose of this paper is to introduce these integers $c_{\lambda}(r)$, which we regard as generalized Littlewood-Richardson coefficients. We have a conjectured formula for $c_{\lambda}(r)$ as the number of sequences $\left(T_{1}, T_{2}, \ldots, T_{n}\right)$ of Young tableaux, with $T_{i}$ of shape $\lambda(i)$, satisfying certain conditions. This formula has been proved when the number of bundles is at most four, but it appears to be a difficult combinatorial problem to prove it in general.

In [8] a special case of this situation was studied, where the rank conditions are given by a permutation $w$. For maps

$$
G_{1} \rightarrow G_{2} \rightarrow \cdots \rightarrow G_{m} \rightarrow F_{m} \rightarrow F_{m-1} \rightarrow \cdots \rightarrow F_{1}
$$

with $\operatorname{rank}\left(G_{i}\right)=\operatorname{rank}\left(F_{i}\right)=i$, and $w \in S_{m+1}$, let

$$
\Omega_{w}=\left\{x \in X \mid \operatorname{rank}\left(G_{q}(x) \rightarrow F_{p}(x)\right) \leq r_{w}(p, q) \forall p, q \leq m\right\},
$$

where $r_{w}(p, q)=\#\{i \leq p \mid w(i) \leq q\}$. These loci are special cases of the loci $\Omega_{r}$ described in this paper. The formulas given here therefore specialize to the universal double Schubert polynomials $\mathfrak{S}_{w}\left(c_{\bullet}\left(F_{\bullet}\right) ; c_{\bullet}\left(G_{\bullet}\right)\right)$ for these loci. Since these universal Schubert polynomials specialize to quantum and double Schubert polynomials ([5], [13, [7]), we derive formulas for these important polynomials. These formulas appear to be new even for the single Schubert polynomials $\mathfrak{S}_{w}(x)$.

Among the loci considered here are the varieties of complexes, which are the loci $\Omega_{r}$ with $r_{i j}=0$ for $j-i \geq 2$. In this case the formula for the coefficients $c_{\lambda}(r)$ is particularly simple, and it agrees with our general conjectured formula.

In Section 2 we discuss the loci $\Omega_{r}$ in more detail, state the main theorem, and derive the main applications. This includes a precise statement of what it means for a polynomial $\sum c_{\lambda}(r) s_{\lambda}\left(E_{\bullet}\right)$ to give a formula for a locus $\Omega_{r}$. This statement implies the assertion that if $X$ is non-singular and $\Omega_{r}$ has the expected codimension $d(r)$, then

$$
\left[\Omega_{r}\right]=\sum_{\lambda} c_{\lambda}(r) s_{\lambda}\left(E_{\bullet}\right)
$$

in the Chow group $A^{d(r)}(X)$. However, weaker assertions can be made when $X$ is singular or the maps $\phi_{i}$ are less generic. At the end of Section 3 we sketch a generalization, which is based on explicit resolutions of singularities of these loci.

The coefficients $c_{\lambda}(r)$ are determined by the geometry, if this assertion is interpreted correctly. We will see in Section 2 that $c_{\lambda}(r)$ depends only on the differences $r_{i, j-1}-r_{i j}$ and $r_{i+1, j}-r_{i j}$. This allows the ranks of the bundles $E_{i}$ to be taken large compared to the expected codimension $d(r)$; if the Chern classes of the bundles are independent, the coefficients $c_{\lambda}(r)$ are then uniquely determined by (1.4). 
Much of the work in a project of this kind - discovering the shape of the formulais invisible in the final product, which has a short proof (given in Section 3). In particular, it came as a pleasant surprise to us that the polynomials for all the loci $\Omega_{r}$ can be written as a linear combination of the polynomials $s_{\lambda}\left(E_{\bullet}\right)$. We know of no reason for this other than the proof of the explicit formula. That the coefficients $c_{\lambda}(r)$ appear to be non-negative is even more surprising.

The conjectured formula for the coefficients is discussed in more detail in the final Section 4 ; proofs of the combinatorial assertions made there can be found in 4.

We are particularly grateful to S. Fomin, who provided an involution on pairs of tableaux which gave us the strongest evidence for the conjectured formula, and who has collaborated with us on the combinatorial aspects of this problem. Thanks also to M. Haiman and M. Shimozono for their responses to combinatorial questions. The Schubert package [12] was useful for calculations.

\section{QUIVER VARIETIES; THE THEOREM AND APPLICATIONS}

2.1. The Main Theorem. Given vector bundles $E_{0}, \ldots, E_{n}$ on a variety $X$, let $H$ be the direct sum of the bundles $\operatorname{Hom}\left(E_{i-1}, E_{i}\right)$, i.e.

$$
H=\operatorname{Hom}\left(E_{0}, E_{1}\right) \times_{X} \operatorname{Hom}\left(E_{1}, E_{2}\right) \times_{X} \cdots \times_{X} \operatorname{Hom}\left(E_{n-1}, E_{n}\right) .
$$

Writing $\widetilde{E}_{i}$ for the pullback of $E_{i}$ to $H$, we have a universal or tautological sequence of bundle maps

$$
\widetilde{E}_{0} \stackrel{\Phi_{1}}{\longrightarrow} \widetilde{E}_{1} \stackrel{\Phi_{2}}{\longrightarrow} \widetilde{E}_{2} \rightarrow \cdots \rightarrow \widetilde{E}_{n-1} \stackrel{\Phi_{n}}{\longrightarrow} \widetilde{E}_{n}
$$

on $H$. For this universal case, it is a theorem of Lakshmibai and Magyar 14 that for $r$ satisfying (1.2), the scheme $\widetilde{\Omega}_{r}=\Omega_{r}\left(\widetilde{E}_{\bullet}\right)$ for (2.1) is reduced and irreducible, of codimension $d(r)$. Moreover, $\widetilde{\Omega}_{r}$ is a Cohen-Macaulay variety if $X$ is CohenMacaulay. (Earlier Abeasis, del Fra, and Kraft [2] had shown, in characteristic zero, that the reduced scheme $\left(\widetilde{\Omega}_{r}\right)_{\text {red }}$ is Cohen-Macaulay.) Note that, when the bundles are trivial, $H$ is a Cartesian product of $X$ and a product $M$ of spaces of matrices, and $\widetilde{\Omega}_{r}$ is a product of $X$ with the corresponding locus in $M$; it is this locus in $M$ that is studied in [1], [2], and [14].

The statement that "the polynomial $P=\sum c_{\lambda}(r) s_{\lambda}\left(E_{\text {• }}\right)$ is a formula for the locus $\Omega_{r}$ " has the usual meaning in intersection theory (cf. [6, §14], [10, App. A]). It implies that when $X$ is non-singular and $\operatorname{codim}\left(\Omega_{r}, X\right)=d(r)$, then

$$
\left[\Omega_{r}\right]=\sum_{\lambda} c_{\lambda}(r) s_{\lambda}\left(E_{\bullet}\right)
$$

in the Chow group $A^{d(r)} X$, where $\left[\Omega_{r}\right]$ is the cycle defined by the scheme $\Omega_{r}$. For arbitrary $X$ and maps $\phi_{i}$, there is a well defined cycle class $\Omega_{r}$ in the Chow group $A_{m-d(r)}\left(\Omega_{r}\right)$, where $m=\operatorname{dim}(X)$, whose image in $A_{m-d(r)}(X)$ is the class $\sum c_{\lambda}(r) s_{\lambda}\left(E_{\bullet}\right) \cap[X]$. Whenever $\Omega_{r}$ has codimension $d(r)$ in $X, \boldsymbol{\Omega}_{r}$ is a positive cycle supported on $\Omega_{r}$; if $X$ is Cohen-Macaulay, or more generally if $\operatorname{depth}\left(\Omega_{r}, X\right)=d(r)$, this cycle is $\left[\Omega_{r}\right]$, but if $X$ is not Cohen-Macaulay the coefficient of a component of $\Omega_{r}$ in $\boldsymbol{\Omega}_{r}$ may be smaller than the length of $\Omega_{r}$ at its generic point. These classes $\boldsymbol{\Omega}_{r}$ are compatible with the basic constructions of intersection theory, exactly as in [6. Thm. 14.3]. 
In fact, to give maps $\phi_{i}: E_{i-1} \rightarrow E_{i}$ for all $i$ is the same as giving a section $s: X \rightarrow H$ of the bundle $H$, and $\Omega_{r}=s^{-1}\left(\widetilde{\Omega}_{r}\right)$. The general class $\boldsymbol{\Omega}_{r}$ is constructed by intersecting $\widetilde{\Omega}_{r} \subset H$ with the (regular) embedding $s: X \rightarrow H$, i.e.

$$
\boldsymbol{\Omega}_{r}=s^{!}\left[\widetilde{\Omega}_{r}\right]
$$

where $s^{!}: A_{*}\left(\widetilde{\Omega}_{r}\right) \rightarrow A_{*}\left(\Omega_{r}\right)$ is the refined intersection $[6, \S 6]$. As in $\left.\sqrt{6}, \S 14\right]$, the general properties of these classes follow from this construction. It therefore suffices to prove the corresponding formula on $H$, i.e. that

$$
\left[\widetilde{\Omega}_{r}\right]=\sum_{\lambda} c_{\lambda}(r) s_{\lambda}\left(\widetilde{E}_{\bullet}\right) \cap[H]
$$

in $A_{N-d(r)}(H)$, where $N=\operatorname{dim}(H)=\operatorname{dim}(X)+\sum_{i=1}^{n} e_{i-1} e_{i}, e_{i}=\operatorname{rank}\left(E_{i}\right)$.

It is natural to arrange the rank conditions in a triangular array:

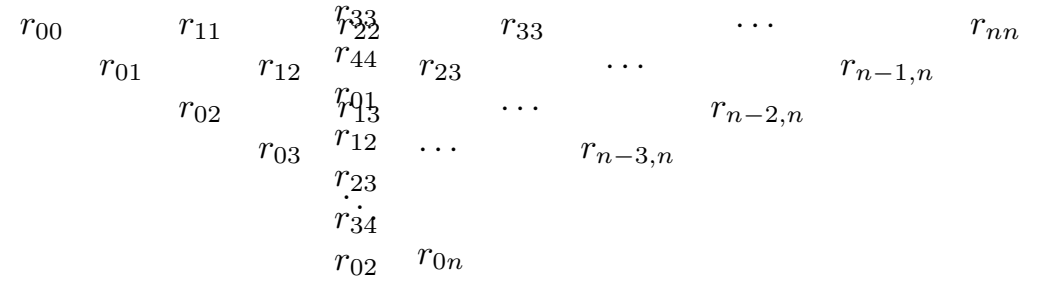

It is useful to replace each small triangle

$$
r^{e} \quad f
$$

occurring in this array by the rectangle of width $e-r$ and height $f-r$.

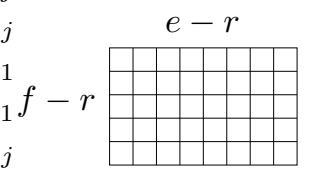

We then have the rectangular array

$$
\begin{array}{ccccccccc}
R_{01} & & R_{12} & & R_{23} & & \ldots & & R_{n-1, n} \\
& R_{02} & & R_{13} & & \ldots & & R_{n-2, n} & \\
& & R_{03} & & \ldots & & R_{n-3, n} & & \\
& & \ddots & & & & & \\
& & & & R_{0 n} & & & &
\end{array}
$$

where $R_{i j}$ has width $r_{i, j-1}-r_{i j}$ and height $r_{i+1, j}-r_{i j}$. Note that the expected codimension $d(r)$ is the sum of the areas of the rectangles. The condition (1.2) says that the rectangles get (weakly) shorter as one proceeds in a southeasterly direction, and they get (weakly) narrower as one travels southwest. For example, the rank conditions given in the triangular array

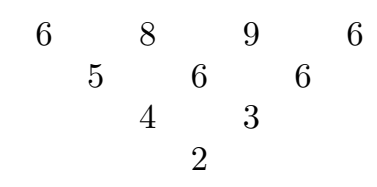


correspond to the rectangular array:

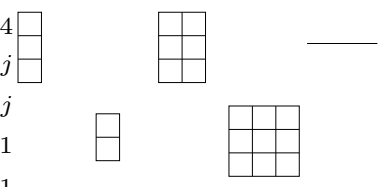

Our formula depends on the rectangles in this array. To be precise, it depends on the integers $r_{i, j-1}-r_{i j}$ and $r_{i+1, j}-r_{i j}$ for all $i<j$; if a width $r_{i, j-1}-r_{i j}$ is zero, we need to know the height $r_{i+1, j}-r_{i j}$, even though the rectangle $R_{i j}$ is empty. (The conjectured formula discussed later does not have this defect.) Each $R_{i j}$ is identified with the partition $\left(r_{i, j-1}-r_{i j}\right)^{r_{i+1, j}-r_{i j}}$ for which it is the Young diagram.

At this point we need some notation. If $R$ is a rectangle of width $e$ and height $f$, and $\sigma$ and $\tau$ are partitions, with the length $\ell(\sigma)$ of $\sigma$ at most $f$, then $(R+\sigma, \tau)$ denotes the sequence $\left(e+\sigma_{1}, e+\sigma_{2}, \ldots, e+\sigma_{f}, \tau_{1}, \tau_{2}, \ldots\right)$; this is a partition only if $e+\sigma_{f} \geq \tau_{1}$. For a partition $\lambda=\left(\lambda_{1}, \ldots, \lambda_{p}\right),|\lambda|$ denotes $\sum \lambda_{i}$, which is the number of boxes in the Young diagram of $\lambda$. For partitions $\lambda, \sigma, \tau$ with $|\sigma|+|\tau|=|\lambda|, c_{\sigma, \tau}^{\lambda}$ denotes the Littlewood-Richardson coefficient, which is the coefficient of the Schur polynomial $s_{\lambda}$ in the expansion of $s_{\sigma} \cdot s_{\tau}$ (see [17]). We set $e_{i}=r_{i i}=\operatorname{rank}\left(E_{i}\right)$, $r_{i}=r_{i-1, i}$, and $R_{i}=R_{i-1, i}$, so $R_{i}$ has height $e_{i}-r_{i}$ and width $e_{i-1}-r_{i}$.

If $I=\left(i_{1}, \ldots, i_{p}\right)$ is a sequence of non-negative integers that is not weakly decreasing, then $s_{I}(F-E)$ is either 0 or it is $\pm s_{\lambda}(F-E)$ for some unique partition $\lambda$ and unique coefficient \pm 1 . This partition and coefficient can be found by performing a sequence of moves of the type

$$
\left(j_{1}, \ldots, j_{p}\right) \mapsto\left(j_{1}, \ldots, j_{k-1}, j_{k+1}-1, j_{k}+1, j_{k+2}, \ldots, j_{p}\right)
$$

if $j_{k+1}>j_{k}$; if one reaches a sequence $\left(j_{1}, \ldots, j_{p}\right)$ with some $j_{k+1}=j_{k}+1$, then $s_{I}=0$; otherwise one reaches a partition $\lambda=\left(\lambda_{1}, \ldots, \lambda_{p}\right)$ in $m$ steps, and then $s_{I}=(-1)^{m} s_{\lambda}$.

We now give an algorithm for constructing finite formal sums $\sum c_{\lambda}(r) S(\lambda)$, with $\lambda$ varying over $n$-tuples of partitions $\lambda=(\lambda(1), \ldots, \lambda(n))$. The polynomial for the degeneracy locus $\Omega_{r}$ will be obtained by replacing each $S(\lambda)$ by $s_{\lambda}\left(E_{\bullet}\right)$. In the algorithm we will meet symbols $S(I(1), \ldots, I(n))$ where each $I(j)$ is a sequence of integers. For such symbols we imitate the above rule for Schur polynomials to write $S(I(1), \ldots, I(1))$ as either zero or $\pm S(\lambda(1), \ldots, \lambda(n))$ for unique partitions $\lambda(1), \ldots, \lambda(n)$. If $s_{I(j)}=0$ for any $j$, put $S(I(1), \ldots, I(n))=0$; otherwise write $s_{I(j)}=\epsilon_{j} s_{\lambda(j)}$ for $1 \leq j \leq n$, with $\epsilon_{j}= \pm 1$, and put $S(I(1), \ldots, I(n))=$ $\left(\prod \epsilon_{j}\right) S(\lambda(1), \ldots, \lambda(n))$.

We construct the polynomial $\sum c_{\lambda}(r) S(\lambda)$ by induction on $n$. For $n=1$ we have just one rectangle $R=R_{01}$, and the polynomial is $S(R)$, which gives $s_{R}\left(E_{1}-E_{0}\right)$. Given the rectangular array for $r$, delete the top row. This gives a smaller array, for which we have a polynomial $\sum d_{\mu} S(\mu)$ by induction, the sum over sequences $\mu=(\mu(1), \ldots, \mu(n-1))$ of partitions. The polynomial $\sum c_{\lambda}(r) S(\lambda)$ is obtained by replacing each $S(\mu)$ in $\sum d_{\mu} S(\mu)$ by

$$
\sum\left(\prod_{i=1}^{n-1} c_{\sigma(i), \tau(i)}^{\mu(i)}\right) S(I(1), \ldots, I(n)) .
$$


Here the sum is over all sequences $(\sigma(1), \ldots, \sigma(n-1))$ and $(\tau(1), \ldots, \tau(n-1))$ of partitions, with $|\sigma(i)|+|\tau(i)|=|\mu(i)|$, such that the length of $\sigma(i)$ is at most the height of $R_{i}$, i.e. $\ell(\sigma(i)) \leq e_{i}-r_{i}$. Define $I(i)$ to be $\left(R_{i}+\sigma(i), \tau(i-1)\right)$ for $1 \leq i \leq n$, where $\tau(0)$ and $\sigma(n)$ are taken to be the empty partition. One uses the rules just given to write each $S(I(1), \ldots, I(n))$ as 0 or $\pm S(\lambda)$ for a unique $\lambda=(\lambda(1), \ldots, \lambda(n))$, thus arriving at a polynomial $\sum c_{\lambda}(r) S(\lambda)$.

Main Theorem. The formula for $\Omega_{r}$ is $\sum c_{\lambda}(r) s_{\lambda}\left(E_{\bullet}\right)$.

This theorem will be proved in the next section. We first interpret it in the case where the rectangular array has only two non-empty rows, i.e. $R_{i j}=\emptyset$ if $j-i>2$. In this case the inductive polynomial $\sum d_{\mu} S(\mu)$ is just $S(\mu)$, for $\mu=\left(R_{02}, R_{13}, \ldots, R_{n-2, n}\right)$. For a rectangle shape $R$, the Littlewood-Richardson coefficient $c_{\sigma, \tau}^{R}$ vanishes unless $\sigma$ and the $180^{\circ}$ rotation of $\tau$ fit together to make $R$, in which case $c_{\sigma, \tau}^{R}=1$.

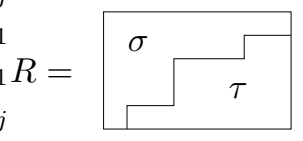

Corollary 1. If $R_{i j}$ is empty for $j-i>2$, then the formula for $\Omega_{r}$ is $\sum s_{\lambda}\left(E_{\mathbf{0}}\right)$, where the sum is over all $\lambda=(\lambda(1), \ldots, \lambda(n))$, with $\lambda(i)=\left(R_{i}+\sigma(i), \tau(i-1)\right)$, such that $\sigma(i)$ and $\tau(i)$ fit together to form $R_{i-1, i+1}$ for $1 \leq i \leq n-1$; here $\sigma(n)$ and $\tau(0)$ are empty.

Note that, by (1.2), for any division of $R_{i-1, i+1}$ into $\sigma(i)$ and $\tau(i), \sigma(i)$ always fits on the right side of $R_{i}$, and $\tau(i)$ fits below $R_{i+1}$, so the resulting sequences $\lambda(i)=\left(R_{i}+\sigma(i), \tau(i-1)\right)$ are always partitions. This formula can be remembered by the picture

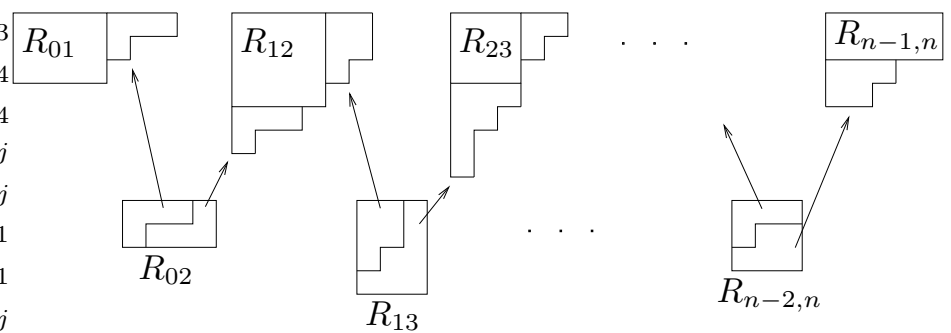

The situation in the corollary covers the case of varieties of complexes, which means that $r_{i j}=0$ for $j-i \geq 2$. In this case the triangular array is

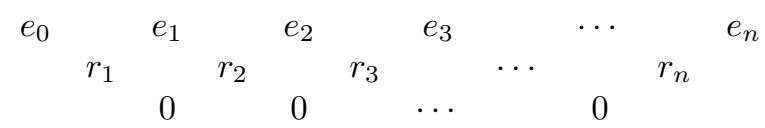


so the array of rectangles is

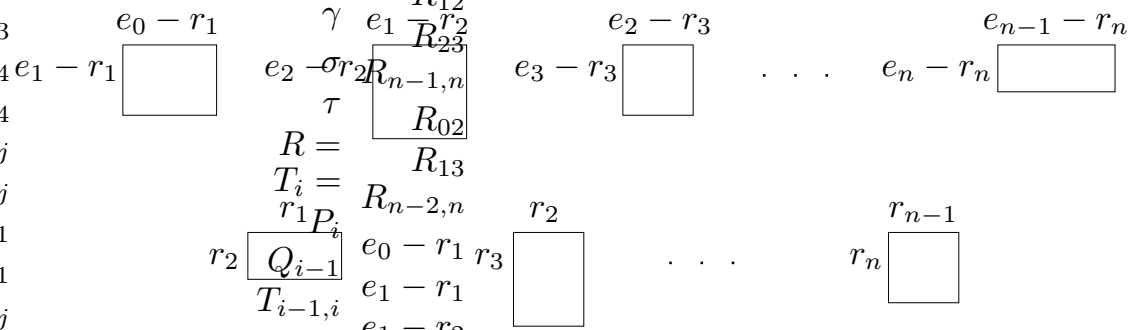

P. Pragacz reports that he had known this formula for $\Omega_{r}$ in the case of varieties of complexes.

2.2. Geometric description of $c_{\lambda}(r)$. Although we use the notation $c_{\lambda}(r)$ for the coefficients, it should be emphasized that they depend only on the differences $r_{i, j-1}-r_{i j}$ and $r_{i+1, j}-r_{i j}$, not on the integers $r_{i j}$ themselves. For example they are unchanged if the same positive integer is added to each $r_{i j}$.

The linear independence of ordinary Schur polynomials $s_{\lambda}\left(x_{1}, \ldots, x_{p}\right)$ as $\lambda$ varies over partitions of length at most $p$, implies that the polynomials $s_{\lambda}\left(E_{\bullet}\right)$ are linearly independent functions of the Chern classes of the bundles $E_{0}, E_{1}, \ldots, E_{n}$, if the ranks of the bundles are suitably large (e.g. if $\ell(\lambda(i)) \leq e_{i}$ for $1 \leq i \leq n$ ).

From the preceding two paragraphs it follows that the coefficients $c_{\lambda}(r)$ are uniquely determined by the geometry, i.e. by the fact that $\sum c_{\lambda}(r) s_{\lambda}\left(E_{\bullet}\right)$ is a formula for $\Omega_{r}$. To see this, one can choose the ranks $e_{i}$ large, and one can find a smooth variety $X$ on which $\Omega_{r}$ has the expected codimension $d(r)$, and for which the classes $s_{\lambda}\left(E_{\bullet}\right)$, for $\sum|\lambda(i)|=d(r)$, are linearly independent. For example, one can start with universal bundles $E_{i}$ on large Grassmanians $G_{i}$, let $G=\prod_{i=0}^{n} G_{i}$, and set $X=\oplus_{i=1}^{n} \operatorname{Hom}\left(E_{i-1}, E_{i}\right)$.

2.3. Schubert polynomials. In [1], the rank conditions $r$ satisfying (1.2) are described by diagrams of dots connected by lines. One puts $e_{i}=r_{i i}$ dots in column $i$, and lines are drawn between some dots in adjacent columns. Then $r_{i j}$ is the number of lines connecting a dot in column $i$ to a dot in column $j$. The example given earlier in this section can be described by the diagram

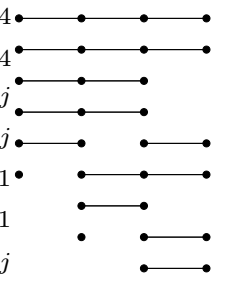

Now fix a positive integer $m$. For a permutation $w \in S_{m+1}$, we form a diagram with $2 m$ columns of lengths $1,2, \ldots, m, m, m-1, \ldots, 2,1$. All possible lines are drawn among the first $m$ columns and among the last $m$ columns. Between the two middle columns, the $i$ 'th dot on the right is connected to the $w(i)$ 'th dot on the left. If $w(i)=m+1$, no connection is made. For example, if $m=4$ and $w=31452$, this diagram is

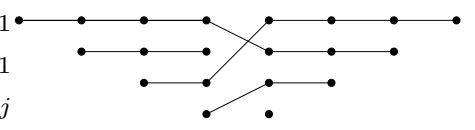


The number of connections between the left column with $q$ dots and the right column with $p$ dots is the number

$$
r_{w}(p, q)=\#\{i \leq p \mid w(i) \leq q\} .
$$

There are the maximal number of connections between two columns on the left or between two on the right. This means that for a sequence $E_{\text {. }}$ of bundle maps

$$
G_{1} \rightarrow G_{2} \rightarrow \cdots \rightarrow G_{m} \rightarrow F_{m} \rightarrow F_{m-1} \rightarrow \cdots \rightarrow F_{1}
$$

with $\operatorname{rank}\left(G_{i}\right)=\operatorname{rank}\left(F_{i}\right)=i$, the locus $\Omega_{r}\left(E_{\bullet}\right)$ defined in the introduction is exactly the locus $\Omega_{w}$ defined in [8], with the same scheme structure. In [8] "universal Schubert polynomials" $\mathfrak{S}_{w}\left(c_{\bullet}\left(F_{\bullet}\right) ; c_{\bullet}\left(G_{\bullet}\right)\right)$ were constructed, which represent the loci $\Omega_{w}$. From the fact that the formula for a locus is unique, we deduce the following corollary.

Corollary 2. With $r$ determined by $w$ as above,

$$
\mathfrak{S}_{w}\left(c_{\bullet}\left(F_{\bullet}\right) ; c_{\bullet}\left(G_{\bullet}\right)\right)=\sum_{\lambda} c_{\lambda}(r) s_{\lambda}\left(E_{\bullet}\right)
$$

When these bundle maps are specialized so that each $G_{i-1} \rightarrow G_{i}$ is an inclusion of bundles, and each $F_{i} \rightarrow F_{i-1}$ is a surjection, then $\mathfrak{S}_{w}\left(c_{\bullet}\left(F_{\bullet}\right) ; c_{\bullet}\left(G_{\bullet}\right)\right)$ becomes the double Schubert polynomial

$$
\mathfrak{S}_{w}\left(x_{1}, \ldots, x_{m} ; y_{1}, \ldots, y_{m}\right)
$$

of Lascoux and Schützenberger; here we set $x_{i}=c_{1}\left(\operatorname{ker}\left(F_{i} \rightarrow F_{i-1}\right)\right)$ and $y_{i}=$ $c_{1}\left(G_{i} / G_{i-1}\right)$. The right side of the formula in this corollary also simplifies in this case. It follows from the definition that for a partition $\tau$, we have

$$
s_{\tau}\left(G_{i}-G_{i-1}\right)= \begin{cases}\left(y_{i}\right)^{q} & \text { if } \tau=(q), q \geq 0 \\ 0 & \text { otherwise }\end{cases}
$$

and

$$
s_{\tau}\left(F_{i-1}-F_{i}\right)= \begin{cases}\left(-x_{i}\right)^{p} & \text { if } \tau=(1)^{p}, p \geq 0 \\ 0 & \text { otherwise. }\end{cases}
$$

Thus $s_{\lambda}\left(E_{\bullet}\right)=0$ unless $\lambda=\left(\left(q_{2}\right),\left(q_{3}\right), \ldots,\left(q_{m}\right), \tau,(1)^{p_{m}}, \ldots,(1)^{p_{2}}\right)$, in which case

$$
s_{\lambda}\left(E_{\bullet}\right)=(-1)^{p_{2}+\cdots+p_{m}} x_{2}^{p_{2}} \cdots x_{m}^{p_{m}} y_{2}^{q_{2}} \cdots y_{m}^{q_{m}} s_{\tau}(x / y),
$$

where $s_{\tau}(x / y)=\operatorname{det}\left(h_{\tau_{i}+j-i}\right), \sum h_{k}=\prod\left(1-y_{i}\right) / \prod\left(1-x_{j}\right)$. Our formula therefore writes $\mathfrak{S}_{w}(x ; y)$ as a signed sum of monomials in the $x_{2}, \ldots, x_{m}, y_{2}, \ldots, y_{m}$ times Schur polynomials $s_{\tau}(x / y)$. When all variables $y_{i}$ are set equal to zero, we have only the terms with $q_{2}=\cdots=q_{m}=0$, and this writes the ordinary Schubert polynomial $\mathfrak{S}_{w}(x)=\mathfrak{S}_{w}(x ; 0)$ as a signed sum of monomials in $x_{2}, \ldots, x_{m}$ times (symmetric) Schur polynomials $s_{\tau}(x)$.

Unlike the inductive construction of Schubert polynomials from high degree to low degree, our formulas are simplest for those of low degree. 
For example, for $w=3142 \in S_{4}$, the corresponding array of rectangles is

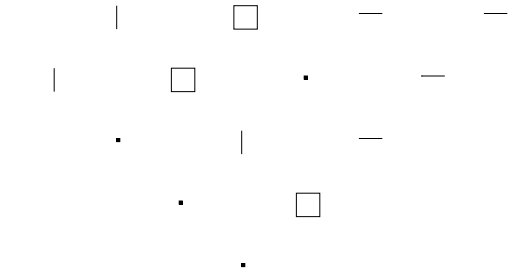

Calculating $\sum c_{\lambda}(r) S(\lambda)$ with the algorithm of the main theorem, working from the bottom up, one has, with $\emptyset$ the empty partition

$$
\begin{aligned}
S(\emptyset) \rightsquigarrow & S(\emptyset, 1) \\
\rightsquigarrow & S(\emptyset, 1, \emptyset)+S(\emptyset, \emptyset, 1) \\
\rightsquigarrow & S(\emptyset, 2, \emptyset, \emptyset)+S(\emptyset, 1,1, \emptyset)+S(\emptyset, 1, \emptyset, 1) \\
\rightsquigarrow & \sum c_{\lambda}(r) S(\lambda)=S(\emptyset, 2,1, \emptyset, \emptyset)+S(\emptyset, 1,11, \emptyset, \emptyset)+ \\
& S(\emptyset, 1,2, \emptyset, \emptyset)+S(\emptyset, 1,1,1, \emptyset)+S(\emptyset, \emptyset, 21, \emptyset, \emptyset)+ \\
& S(\emptyset, \emptyset, 11,1, \emptyset)+S(\emptyset, 1,1, \emptyset, 1)+S(\emptyset, \emptyset, 11, \emptyset, 1) .
\end{aligned}
$$

Substituting $s_{\lambda}\left(E_{\bullet}\right)$ for $S(\lambda)$, this is a formula for the universal double Schubert polynomial associated to $w=3142$. It specializes to the formula

$$
\begin{aligned}
\mathfrak{S}_{3142}(x ; y)=s_{21}(x / y)+y_{3} s_{2}(x / y)+ & \\
& \left(y_{3}-x_{2}-x_{3}\right) s_{11}(x / y)+y_{3}\left(y_{3}-x_{2}-x_{3}\right) s_{1}(x / y)
\end{aligned}
$$

and to

$$
\mathfrak{S}_{3142}(x)=s_{21}(x)-\left(x_{2}+x_{3}\right) s_{11}(x) .
$$

The rectangular array coming from a permutation $w \in S_{m+1}$ has only empty rectangles and $1 \times 1$ rectangles. In fact, this array is determined from the diagram of the permutation denoted $D^{\prime}(w)$ in $[8, \S 2]$ : the diagram $D^{\prime}(w)$ is reflected in a vertical line, then rotated $135^{\circ}$ clockwise, and the result fitted in the bottom of the triangle; each box in $D^{\prime}(w)$ is then in the position of a non-empty rectangle of the rectangular array. In other words the rectangle $R_{i j}$ is non-empty iff $D^{\prime}(w)$ contains a box in position $(2 m-j, i+1)$, which happens exactly when $w(2 m+1-j) \leq i+1$ and $w^{-1}(i+2) \leq 2 m-j$.

\section{Proof of the Theorem}

3.1. Geometric preliminaries. It follows from the general discussion in Section 2 that it suffices to prove the formula for the universal locus $\Omega_{r}\left(E_{\bullet}\right)$ in $H=$ $\bigoplus \operatorname{Hom}\left(E_{i-1}, E_{i}\right)$. (Throughout this section we omit notation for pullbacks of bundles by canonical maps.) In particular, we know from [2] and [14 that $\Omega_{r}\left(E_{\bullet}\right)$ is reduced and irreducible of the expected codimension $d(r)$, and that $\Omega_{r}\left(E_{\bullet}\right)$ is the closure of the locus $\Omega_{r}^{\circ}\left(E_{\bullet}\right)$ where each of the maps $E_{i}(x) \rightarrow E_{j}(x)$ has rank equal to $r_{i j}$ for $i<j$. We must prove that, with these assumptions,

$$
\left[\Omega_{r}\left(E_{\bullet}\right)\right]=\sum_{\lambda} c_{\lambda}(r) s_{\lambda}\left(E_{\bullet}\right) \cap[H]
$$

in the Chow group $A_{N-d(r)}(H), N=\operatorname{dim}(H)$.

Form the Grassmannian bundle $G_{0}$ over $X$ with $r_{i}=r_{i-1, i}$ as in $\$ 2.1$ :

$$
G_{0}=\operatorname{Gr}\left(r_{1}, E_{1}\right) \times_{X} \operatorname{Gr}\left(r_{2}, E_{2}\right) \times_{X} \cdots \times_{X} \operatorname{Gr}\left(r_{n}, E_{n}\right) .
$$


Let $G=G_{0} \times_{X} H$, with projection $\pi: G \rightarrow H$. Let $0 \rightarrow A_{i} \rightarrow E_{i} \rightarrow Q_{i} \rightarrow 0$ be the universal exact sequences on $G_{0}$, and hence also on $G$. Let $Z \subset G$ be the intersection of the zero-schemes of the canonical maps $E_{i-1} \rightarrow E_{i} \rightarrow Q_{i}$, i.e.

$$
Z=Z\left(E_{0} \rightarrow Q_{1}\right) \cap Z\left(E_{1} \rightarrow Q_{2}\right) \cap \cdots \cap Z\left(E_{n-1} \rightarrow Q_{n}\right) .
$$

On $Z$ we have maps $E_{i-1} \rightarrow A_{i}$ for $1 \leq i \leq n$. Composing these with the inclusions $A_{i-1} \subset E_{i-1}$ we get a sequence $A$. of bundles and bundle maps on $Z$ :

$$
A_{1} \rightarrow A_{2} \rightarrow \cdots \rightarrow A_{n}
$$

Let $\bar{r}$ denote the rank conditions obtained by omitting the top row of the triangular array for $r$, and let $\Omega_{\bar{r}}\left(A_{\bullet}\right) \subset Z$ be the locus given by these maps and rank conditions. It is easy to see that $\Omega_{\bar{r}}\left(A_{\bullet}\right)$ is mapped into $\Omega_{r}\left(E_{\bullet}\right)$ by $\pi$.

Now $Z$ is isomorphic to the bundle $\bigoplus_{i=1}^{n} \operatorname{Hom}\left(E_{i-1}, A_{i}\right)$ over $G_{0}$, and we have a canonical projection

$$
\rho: Z=\bigoplus_{i=1}^{n} \operatorname{Hom}\left(E_{i-1}, A_{i}\right) \longrightarrow \bigoplus_{i=2}^{n} \operatorname{Hom}\left(A_{i-1}, A_{i}\right)=H^{\prime} .
$$

Denote by $\Omega^{\prime}$ the universal locus $\Omega_{\bar{r}}\left(A_{\bullet}\right)$ of $H^{\prime}$. Then $\Omega_{\bar{r}}\left(A_{\bullet}\right)$ in $Z$ is the inverse image of $\Omega^{\prime}$ by $\rho$. Since the maps on $H^{\prime}$ are universal, it follows that $\Omega^{\prime}$ is irreducible, and therefore $\Omega_{\bar{r}}\left(A_{\bullet}\right)$ is an irreducible subscheme of $Z$.

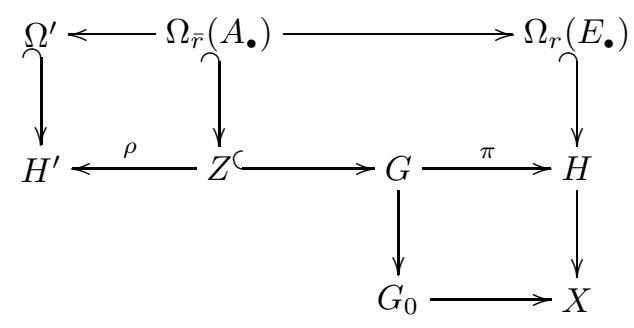

Lemma 1. $\pi$ maps $\Omega_{\bar{r}}\left(A_{\bullet}\right)$ birationally onto $\Omega_{r}\left(E_{\bullet}\right)$.

Proof. Let $Z^{\circ}$ denote the open subset of $Z$ where the maps $E_{i-1} \rightarrow A_{i}$ are surjective. Then the schemes $\Omega_{\bar{r}}^{\circ}\left(A_{\bullet}\right) \cap Z^{\circ}$ and $\Omega_{r}^{\circ}\left(E_{\bullet}\right)$ are isomorphic; they are both universal objects in the category of schemes $Y$ over $H$, such that the pullback to

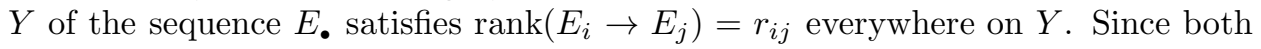
$\Omega_{\bar{r}}\left(A_{\bullet}\right)$ and $\Omega_{r}\left(E_{\bullet}\right)$ are irreducible schemes, and since $\Omega_{r}^{\circ}\left(E_{\bullet}\right) \neq \emptyset$, the assertion follows.

Note that this argument gives a direct proof that the codimension of $\Omega_{r}\left(E_{\boldsymbol{\bullet}}\right)$ in $H$ is $d(r)$. Indeed, by induction we know that $\Omega^{\prime}$ has codimension $d(\bar{r})$ in $H^{\prime}$, and $\Omega_{\bar{r}}\left(A_{\bullet}\right)$ must then have the same codimension in $Z$. We conclude that the codimension of $\Omega_{r}\left(E_{\bullet}\right)$ in $H$ is

$$
d(\bar{r})+\operatorname{dim}(H)-\operatorname{dim}(Z)=d(r) .
$$

3.2. Proof of the main theorem. By induction on $n$ we know that $\left[\Omega^{\prime}\right]=$ $\sum c_{\mu}(\bar{r}) s_{\mu}\left(A_{\bullet}\right) \cap\left[H^{\prime}\right]$, so

$$
\left[\Omega_{\bar{r}}\left(A_{\bullet}\right)\right]=\rho^{*}\left[\Omega^{\prime}\right]=\sum_{\mu} c_{\mu}(\bar{r}) s_{\mu}\left(A_{\bullet}\right) \cap[Z] .
$$


Furthermore $[Z]=\prod_{i=1}^{n} c_{\text {top }}\left(\operatorname{Hom}\left(E_{i-1}, Q_{i}\right)\right) \cap[G]$, so

$$
[Z]=\prod_{i=1}^{n} s_{R_{i}^{\prime}}\left(Q_{i}-E_{i-1}\right) \cap[G]
$$

where $R_{i}^{\prime}=\left(e_{i-1}\right)^{e_{i}-r_{i}}$. Since $\pi_{*}\left[\Omega_{\bar{r}}\left(A_{\bullet}\right)\right]=\left[\Omega_{r}\left(E_{\bullet}\right)\right]$ by Lemma 1, we are therefore reduced to proving the identity

$$
\pi_{*}\left(\sum_{\mu} c_{\mu}(\bar{r}) s_{\mu}\left(A_{\bullet}\right) \cdot \prod_{i=1}^{n} s_{R_{i}^{\prime}}\left(Q_{i}-E_{i-1}\right) \cap[G]\right)=\sum_{\lambda} c_{\lambda}(r) s_{\lambda}\left(E_{\bullet}\right) \cap[H] .
$$

For this we need the following basic Gysin formula of Pragacz [18, Prop. 2.2], whose proof comes from [1], cf. [10, App. F].

Lemma 2. Let $E$ and $F$ be vector bundles of ranks $e$ and $f$ on a variety $X$. Let $0 \leq d \leq \min (e, f)$. Let $G=\operatorname{Gr}(d, F)$ be the Grassmann bundle, with projection $\pi: G \rightarrow X$ and universal exact sequence $0 \rightarrow A \rightarrow F \rightarrow Q \rightarrow 0$. Let $q=f-d$, $R=(e-d)^{q}$, and $R^{\prime}=(e)^{q}$. For any partitions $\lambda$ and $\mu$, with $\lambda$ of length at most $q$, and any $\alpha \in A_{*}(X)$,

$$
\pi_{*}\left(s_{R^{\prime}+\lambda}(Q-E) s_{\mu}(A-E) \cap \pi^{*} \alpha\right)=s_{R+\lambda, \mu}(F-E) \cap \alpha .
$$

We also need the following special case of the factorization formula of Lascoux and Schützenberger [16] and Berele and Reger [3], cf. [18].

Lemma 3. Let $E$ and $F$ be vector bundles of ranks e and $f$. Let $R=(e)^{f}$. Let $\lambda$ be a partition of length at most $f$. Then

$$
s_{\lambda}(F) s_{R}(F-E)=s_{R+\lambda}(F-E) .
$$

Note that this identity follows from Lemma 2. Finally we need the basic identity 17, §1.5]:

Lemma 4. For bundles $E_{1}, E_{2}$, and $E_{3}$, and a partition $\mu$,

$$
s_{\mu}\left(E_{3}-E_{1}\right)=\sum c_{\sigma \tau}^{\mu} s_{\sigma}\left(E_{2}-E_{1}\right) s_{\tau}\left(E_{3}-E_{2}\right),
$$

the sum over partitions $\sigma$ and $\tau$ with $|\sigma|+|\tau|=|\mu|$, with $c_{\sigma \tau}^{\mu}$ the LittlewoodRichardson coefficient.

Now we can prove (3.1). First use Lemma 4 to replace each factor $s_{\mu(i)}\left(A_{i+1}-A_{i}\right)$ that occurs in each $s_{\mu}\left(A_{\bullet}\right)$ on the left side of $(3.1)$ by the sum

$$
\begin{aligned}
\sum c_{\sigma(i), \tau(i)}^{\mu(i)} s_{\sigma(i)}\left(E_{i}-A_{i}\right) s_{\tau(i)}\left(A_{i+1}\right. & \left.-E_{i}\right) \\
& =\sum c_{\sigma(i), \tau(i)}^{\mu(i)} s_{\sigma(i)}\left(Q_{i}\right) s_{\tau(i)}\left(A_{i+1}-E_{i}\right) .
\end{aligned}
$$

Note that $s_{\sigma(i)}\left(Q_{i}\right)=0$ if $\ell(\sigma(i))>\operatorname{rank}\left(Q_{i}\right)=e_{i}-r_{i}$. Next use Lemma 3 to replace each $s_{\sigma(i)}\left(Q_{i}\right) \cdot s_{R_{i}^{\prime}}\left(Q_{i}-E_{i-1}\right)$ in the result by $s_{R_{i}^{\prime}+\sigma(i)}\left(Q_{i}-E_{i-1}\right)$. The left side of (3.1) becomes

$$
\begin{aligned}
\sum_{\mu} c_{\mu}(\bar{r}) \sum_{\sigma(i), \tau(i)} & \left(\prod_{i=1}^{n-1} c_{\sigma(i), \tau(i)}^{\mu(i)}\right) . \\
& \pi_{*}\left(\prod_{i=1}^{n} s_{R_{i}^{\prime}+\sigma(i)}\left(Q_{i}-E_{i-1}\right) s_{\tau(i-1)}\left(A_{i}-E_{i-1}\right) \cap[G]\right) .
\end{aligned}
$$


Finally, $n$ applications of Lemma 2 yields

$$
\begin{aligned}
\pi_{*}\left(\prod _ { i = 1 } ^ { n } s _ { R _ { i } ^ { \prime } + \sigma ( i ) } ( Q _ { i } - E _ { i - 1 } ) s _ { \tau ( i - 1 ) } \left(A_{i}\right.\right. & \left.\left.-E_{i-1}\right) \cap[G]\right) \\
& =\prod_{i=1}^{n} s_{R_{i}+\sigma(i), \tau(i-1)}\left(E_{i}-E_{i-1}\right) \cap[H],
\end{aligned}
$$

and this gives the required formula

$$
\begin{array}{r}
\sum_{\mu} c_{\mu}(\bar{r}) \sum_{\sigma(i), \tau(i)}\left(\prod_{i=1}^{n-1} c_{\sigma(i), \tau(i)}^{\mu(i)}\right) \prod_{i=1}^{n} s_{R_{i}+\sigma(i), \tau(i-1)}\left(E_{i}-E_{i-1}\right) \cap[H] \\
=\sum_{\lambda} c_{\lambda}(r) s_{\lambda}\left(E_{\bullet}\right) \cap[H] .
\end{array}
$$

Although we have stated it for varieties over a field, the theorem (and its proof) extend readily to schemes of finite type over a regular base, as in [6, §20].

3.3. A generalization. There is a generalization of the theorem which may be useful in its own right, and which gives some insight into the proof. (It is not needed in this paper.) Fix $E_{0}, \ldots, E_{n}$ on $X$, and $r=\left(r_{i j}\right)$ satisfying (1.2). Let $H=\bigoplus \operatorname{Hom}\left(E_{i-1}, E_{i}\right)$, on which the tautological bundle maps are universal, and one has the universal locus $\Omega_{r} \subset H$.

Let $\pi: F \rightarrow H$ be the partial flag bundle parameterizing flags in each $E_{j}$ of ranks $r_{0 j}, r_{1 j}, \ldots, r_{j-1, j}$. Let $E_{0 j} \subset E_{1 j} \subset \cdots \subset E_{j-1, j} \subset E_{j}$ denote the tautological flags of vector bundles on $F, 1 \leq j \leq n$.

Let $Z \subset F$ be the locus on which the image of $E_{i, j-1}$ by the map $E_{j-1} \rightarrow E_{j}$ is contained in the subbundle $E_{i j}$, i.e. $Z$ is the subscheme defined by the vanishing of all maps $E_{i, j-1} \rightarrow E_{j} / E_{i j}$ for $i<j$.

One sees as in Lemma 1 that $\pi$ maps $Z$ birationally onto $\Omega_{r}$. In fact, if $X$ is non-singular, this construction gives a canonical resolution of singularities of the universal locus $\Omega_{r}$. It is easy to see that the class of $Z$ is given by

$$
[Z]=\prod_{i<j} z_{i j}
$$

where $z_{i j}=c_{\text {top }}\left(\operatorname{Hom}\left(E_{i, j-1}, E_{i+1, j} / E_{i j}\right)\right)$.

Consider a path $\gamma$ through the triangular array for $r$, going from $r_{00}$ to $r_{n n}$. The path must be a union of line segments between neighboring rank conditions, and it must intersect any vertical line at most once.

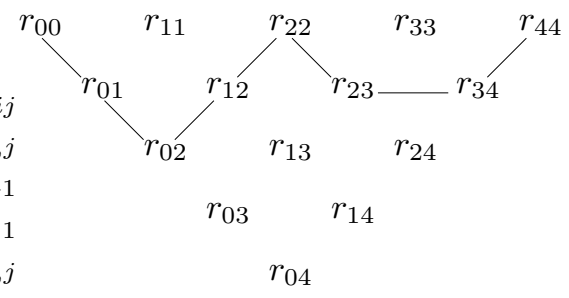

For each $j=1, \ldots, n$, let $k_{j}$ be minimal such that $\gamma$ goes through $r_{k_{j}, j}$. Let $F(\gamma)$ be the partial flag bundle parameterizing flags in $E_{j}$ of ranks $r_{k_{j}, j}, r_{k_{j}+1, j}, \ldots, r_{j-1, j}$, and let $E_{k_{j}, j} \subset \cdots \subset E_{j-1, j} \subset E_{j}$ be the tautological flags on $F(\gamma), 1 \leq j \leq n$. If the path has $m$ line segments, we let $A_{0}, A_{1}, \ldots, A_{m}$ denote the vector bundles on 
$F(\gamma)$ corresponding to the rank conditions passed through by the path. (In the illustration, $m=7$, and the bundle sequence is $E_{00}, E_{01}, E_{02}, E_{12}, E_{22}, E_{23}, E_{34}, E_{44}$.)

Let $\Omega_{r}(\gamma) \subset F(\gamma)$ be the subscheme defined by the conditions that each map $E_{i, j-1} \rightarrow E_{j} / E_{i j}$ vanishes for $r_{i j}$ on or above the path, and $\operatorname{rank}\left(E_{i p} \rightarrow E_{j}\right) \leq r_{i j}$ for $r_{i p}$ on or above the path and $p \leq j$. The canonical maps $F \rightarrow F(\gamma) \rightarrow X$ map $Z$ birationally onto $\Omega_{r}(\gamma)$ which in turn is mapped birationally onto $\Omega_{r}$. Our goal is to give a formula for the class of $\Omega_{r}(\gamma)$. To do this, we define a formal linear combination $\Phi(\gamma)$ of symbols $S(\lambda)$, where $\lambda$ is a sequence of partitions, one for each line segment in $\gamma$. The formula for $\Omega_{r}(\gamma)$ is obtained by replacing each $S(\lambda(1), \ldots, \lambda(m))$ by $\prod_{i=1}^{m} s_{\lambda(i)}\left(A_{i}-A_{i-1}\right)$, and multiplying the result by $\prod z_{i j}$, the product over all $i, j$ such that $r_{i j}$ is on or above $\gamma$.

We define $\Phi(\gamma)$ inductively. If $\gamma$ is the lowest possible path, going from $r_{00}$ to $r_{0 n}$ to $r_{n n}$, then $\Phi(\gamma)=S(\emptyset, \ldots, \emptyset)$, where the empty partition $\emptyset$ is repeated $2 n$ times. Otherwise, we can find a path $\gamma^{\prime}$ that is equal to $\gamma$ except it goes lower at one place, in one of the following ways:

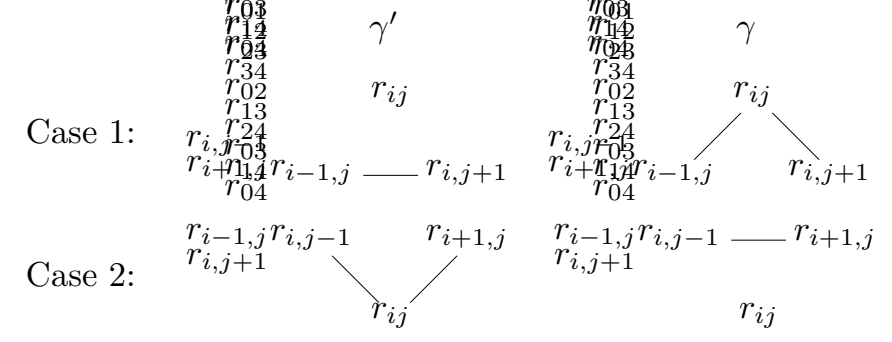

In Case 1 , we obtain $\Phi(\gamma)$ from $\Phi\left(\gamma^{\prime}\right)$ by replacing each symbol $S(\ldots, \mu, \ldots)$ by $\sum c_{\sigma, \tau}^{\mu} S(\ldots, \sigma, \tau, \ldots)$. Note that in this case we have $F(\gamma)=F\left(\gamma^{\prime}\right)$ and $\Omega(\gamma)=$ $\Omega\left(\gamma^{\prime}\right)$. For Case 2, each symbol $S(\ldots, \mu, \nu, \ldots)$ in $\Phi\left(\gamma^{\prime}\right)$ is replaced by the symbol $S\left(\ldots,\left(R_{i j}+\nu, \mu\right), \ldots\right)$. Note that the partitions $\lambda(i)$ are always empty for line segments on the left or right edges of the triangle.

The proof that this polynomial gives a formula for $\left[\Omega_{r}(\gamma)\right]$ is similar to that of our main theorem: one shows that $[Z]$ pushes forward to the class of this polynomial. The induction step, in either Case 1 or 2 , is more transparent, as changes are made in only one segment of the formula.

If bundle maps and flags of subbundles are given on $X$ corresponding to ranks on or above the path $\gamma$, these determine a section $s: X \rightarrow F(\gamma)$, so one has corresponding formulas for the classes $s^{*}\left[\Omega_{r}(\gamma)\right]$. When $\gamma$ is the horizontal path across the top of the diagram, we recover the main theorem.

\section{ON THE COEFFICIENTS $c_{\lambda}(r)$}

There are some properties of the coefficients $c_{\lambda}(r)$ that follow from geometry, i.e. from the main theorem, although they are not obvious from the algorithm defining them. We describe these first, and then discuss properties we believe for other reasons. We conclude with a comparison of the numbers $c_{\lambda}(r)$ with LittlewoodRichardson coefficients.

Consider the dual sequence

$$
E_{n}^{\vee} \rightarrow E_{n-1}^{\vee} \rightarrow \cdots \rightarrow E_{0}^{\vee}
$$

with dual rank conditions, $\operatorname{rank}\left(E_{j}^{\vee} \rightarrow E_{i}^{\vee}\right) \leq r_{i j}$, which we denote by $r^{\vee}$; then $\Omega_{r} \vee\left(E_{\bullet}^{\vee}\right)=\Omega_{r}\left(E_{\bullet}\right)$. Note that the rectangular array for $r^{\vee}$ is obtained by reflecting 
that for $r$ in a vertical line, and replacing each rectangle by its transpose. Using the basic identity that $s_{\lambda}(F-E)=s_{\lambda^{\prime}}\left(E^{\vee}-F^{\vee}\right)$, where $\lambda^{\prime}$ is the transpose of $\lambda$, we find that

$$
c_{\lambda \vee}\left(r^{\vee}\right)=c_{\lambda}(r)
$$

where, if $\lambda=(\lambda(1), \ldots, \lambda(n))$, we put $\lambda^{\vee}=\left(\lambda(n)^{\prime}, \ldots, \lambda(1)^{\prime}\right)$.

It can happen that for some $k$, all of the rank conditions $\operatorname{rank}\left(E_{i} \rightarrow E_{k}\right) \leq r_{i k}$ and $\operatorname{rank}\left(E_{k} \rightarrow E_{j}\right) \leq r_{k j}$ follow from other rank conditions. This happens when, in the rectangle diagram, all the rectangles on the two $45^{\circ}$ lines descending from position $k$ are empty. For the example $G_{1} \rightarrow G_{2} \rightarrow G_{3} \rightarrow F_{3} \rightarrow F_{2} \rightarrow F_{1}$ considered at the end of Section 2, with rank conditions $r$ coming from $w=3142 \in$ $S_{4}$, the bundles $G_{1}$ and $F_{2}$ are inessential in this way. If an inessential bundle $E_{k}$ is omitted, one has a shorter sequence $E_{\bullet}^{\prime}: E_{0} \rightarrow \cdots \rightarrow E_{k-1} \rightarrow E_{k+1} \rightarrow \cdots \rightarrow E_{n}$, with the map $E_{k-1} \rightarrow E_{k+1}$ being $\phi_{k+1} \circ \phi_{k}$, and corresponding rank conditions $r^{\prime}$; the array of rectangles for $r^{\prime}$ is obtained by omitting the $45^{\circ}$ lines of empty rectangles and moving all rectangles below up a row. For example, if $G_{1}$ and $F_{2}$ are omitted from the example, one has $G_{2} \rightarrow G_{3} \rightarrow F_{3} \rightarrow F_{1}$, with rectangular array

Lemma 4 can be used to expand any $s_{\rho}\left(E_{k+1}-E_{k-1}\right)$ occurring in the formula $\sum c_{\mu}\left(r^{\prime}\right) s_{\mu}\left(E_{\bullet}^{\prime}\right)$ as a sum of terms of the form $s_{\sigma}\left(E_{k}-E_{k-1}\right) \cdot s_{\tau}\left(E_{k+1}-E_{k}\right)$. Since $\Omega_{r^{\prime}}\left(E_{\bullet}^{\prime}\right)=\Omega_{r}\left(E_{\bullet}\right)$, with this interpretation we have

$$
\sum_{\mu} c_{\mu}\left(r^{\prime}\right) s_{\mu}\left(E_{\bullet}^{\prime}\right)=\sum_{\lambda} c_{\lambda}(r) s_{\lambda}\left(E_{\bullet}\right)
$$

Now we turn to our conjectured formula for the coefficients $c_{\lambda}(r)$, which interprets them by counting Young tableaux, in a way similar to and generalizing the classical Littlewood-Richardson rule. Recall that a (semistandard) Young tableau is a filling of the boxes in the Young diagram of a partition with integers that are weakly increasing in rows and strictly increasing down columns. Two Young tableaux $P$ and $Q$ can be multiplied to give another Young tableau denoted $P \cdot Q$. One way to do this is to arrange $P$ and $Q$ corner to corner and play the jeu de taquin, sliding inside corners through but keeping the weak and strict orderings.

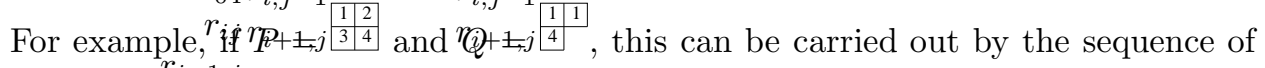
moves

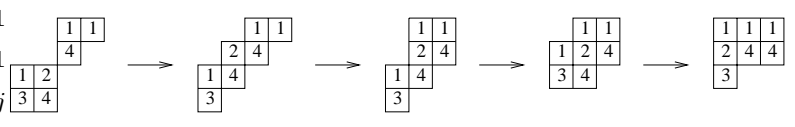

The final tableau is $P \cdot Q$. The main fact is that this product is independent of choice of the sequence of inside corners, from which it follows that the set of tableaux form an associative monoid, called the plactic monoid. With this notion, the Littlewood-Richardson number $c_{\mu, \nu}^{\lambda}$ is the number of ways a given tableau $T$ of shape $\lambda$ can be factored into a product $T=P \cdot Q$, such that $P$ has shape $\mu$ and $Q$ has shape $\nu$. For proofs and relations with Schur polynomials, see [15] or [9].

Given rank conditions $r$ (satisfying (1.2) as always), form the array of rectangles $R_{i j}$. We choose a fixed tableau $T_{i j}$ on each shape $R_{i j}$, with the condition that each 
entry of $T_{i j}$ must be strictly smaller than any entry of $T_{k l}$ if $R_{k l}$ lies in the wedge cut out by $45^{\circ}$ lines below $R_{i j}$, i.e. if $k \leq i$ and $l \geq j$ with $(k, l) \neq(i, j)$.

From this array of rectangular tableaux we will construct a set of $n$-tuples of tableaux $\left(T_{1}, \ldots, T_{n}\right)$ that we call factor sequences. Our conjecture is that $c_{\lambda}(r)$ is the number of factor sequences $\left(T_{1}, \ldots, T_{n}\right)$ such that $T_{i}$ has shape $\lambda(i)$ for $1 \leq i \leq n$. We first explain this for $n=3$, where we start with an array of rectangular tableaux:

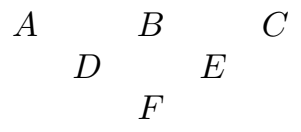

Factor $F$ into a product $F=F_{1} \cdot F_{2}$ of tableaux. Pass $F_{1}$ up to the left, and multiply it to $D$ from the right. Pass $F_{2}$ up to the right and multiply it to $E$ from the left. Then factor the results:

$$
D \cdot F_{1}=D_{1} \cdot D_{2} \quad \text { and } \quad F_{2} \cdot E=E_{1} \cdot E_{2} .
$$

Pass the results up to the left and right, arriving at tableaux $A \cdot D_{1}, D_{2} \cdot B \cdot E_{1}$, and $E_{2} \cdot C$. This gives a factor sequence $\left(T_{1}, T_{2}, T_{3}\right)=\left(A \cdot D_{1}, D_{2} \cdot B \cdot E_{1}, E_{2} \cdot C\right)$.

In general one proceeds by induction. A factor sequence for the given array of rectangular tableaux is obtained by forming a factor sequence $\left(S_{1}, \ldots, S_{n-1}\right)$ for the array of the bottom $n-1$ rows. Factor each $S_{i}$ arbitrarily into $S_{i}=P_{i} \cdot Q_{i}$. Then

$$
\left(T_{1}, \ldots, T_{n}\right)=\left(T_{01} \cdot P_{1}, \ldots, Q_{i-1} \cdot T_{i-1, i} \cdot P_{i}, \ldots, Q_{n-1} \cdot T_{n-1, n}\right)
$$

is a factor sequence for the given array.

Conjecture. $c_{\lambda}(r)$ is the number of factor sequences $\left(T_{1}, \ldots, T_{n}\right)$ of shape $\lambda=$ $(\lambda(1), \ldots, \lambda(n))$ that can be made from a given array of rectangular tableaux.

The conjecture has a number of consequences:

C1. Each $c_{\lambda}(r)$ is a non-negative integer.

C2. The coefficients $c_{\lambda}(r)$ depend only on the rectangles $R_{i j}$, not on their sides.

This means that if one of the sides of a rectangle $R_{i j}$ is 0 , the length of the other side is irrelevant. (The algorithm of the main theorem shows this when the height of a rectangle is 0 , but not when the width is 0 .)

Implicit in the conjecture is the assertion

C3. The number of factor sequences of shape $\lambda$ is independent of choice of fixed tableaux $T_{i j}$.

Granting C3, it is not hard to see that the conjectured formula for the $c_{\lambda}(r)$ satisfies the duality (4.1). For this one chooses the $T_{i j}$ so that no entry appears more than once, and uses the fact that factoring a tableau $T$ with distinct entries into $P \cdot Q$ is equivalent to factoring its conjugate $T^{\prime}$ into $Q^{\prime} \cdot P^{\prime}$. It is also not difficult to verify that the conjectured formula satisfies the property (4.2) for omitting inessential bundles.

The conjecture is true for the case where $R_{i j}$ is empty for $j-i>2$. This follows from the description in Corollary 1 of Section 2, together with the fact that for a tableau $T$ of rectangular shape $R$, for each $\sigma$ and $\tau$ that fit together to form $R$, there is a unique factorization $T=P \cdot Q$ with $P$ of shape $\sigma$ and $Q$ of shape $\tau$; conversely, any factorization of $T$ has factors of shapes that fit together to form $R$. 
The conjecture has been proved when $n \leq 3$. More generally, it is proved when $R_{i j}$ is empty for $j-i>3$ and no two non-empty rectangles in the third row are adjacent. The proof depends on a wonderful involution on pairs of tableaux produced for us by S. Fomin. This proof is given in [4].

One reason that the combinatorial formula is hard to work with is that a given factor sequence can arise in many ways by the algorithm that produces them. At first glance it would appear that to tell if some $\left(T_{1}, \ldots, T_{n}\right)$ is a factor sequence, one would have to test all possible ways of carrying out the sequence of factorings. However, there is a direct test. For this, define $P_{i}$ to be the part of $T_{i}$ lying to the right of the rectangle $R_{i}$, and define $Q_{i-1}$ to be everything lying below $T_{i-1, i}$ and $P_{i}$ :

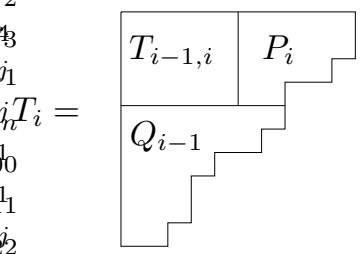

Then $\left(T_{1}, \ldots, T_{n}\right)$ is a factor sequence if and only if $Q_{0}$ and $P_{n}$ are empty and $\left(P_{1} \cdot Q_{1}, \ldots, P_{n-1} \cdot Q_{n-1}\right)$ is a factor sequence for the lower $n-1$ rows of the array. By induction this gives a direct algorithm to test, from the top down. Note that this algorithm, like the theorem, uses the height of a rectangle $R_{i j}$ even if its width is zero. This criterion is proved in [4].

The full conjecture follows from an assertion that Fomin's involution preserves factor sequences. This assertion is true for $n \leq 3$, and it has been verified in 500,000 randomly generated examples for $n \leq 10$. For a discussion of Fomin's involution and the discussion of this, we refer again to [ 4 .

The numbers $c_{\lambda}(r)$ generalize Littlewood-Richardson numbers $c_{\alpha, \beta}^{\gamma}$ in fact as well as in spirit. To see this, take any rectangle $R$ containing $\gamma$, and let $\tau$ be the complement of $\gamma$ in $R$ (rotated $180^{\circ}$ ):

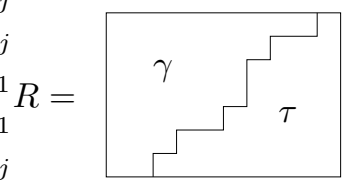

Form the array of rectangles

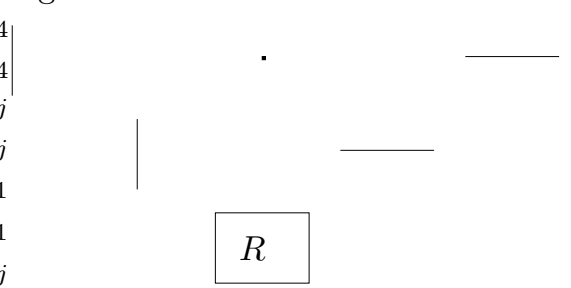

where the vertical lines are the height of $R$, the horizontal lines are its width, and the dot is empty. Choose $r$ giving rise to this array. Then

$$
c_{\alpha, \beta}^{\gamma}=c_{\lambda}(r)
$$

with $\lambda=(\alpha, \beta, \tau)$. This follows easily from the theorem (and also from the conjecture). 
There are analogous conjectures for the coefficients of the polynomials for the more general loci described in $\$ 3.3$. In particular, all these coefficients should also be positive. Details will be given in 迎.

\section{REFERENCES}

[1] S. Abeasis and A. Del Fra, Degenerations for the representations of an equioriented quiver of type $A_{m}$, Boll. Un. Mat. Ital. Suppl. 1980, no. 2, 157-171.

[2] S. Abeasis, A. Del Fra, and H. Kraft, The geometry of representations of $A_{m}$, Math. Ann. 256 (1981), 401-418.

[3] A. Berele and A. Regev, Hook Young diagrams, combinatorics and representations of Lie superalgebras, Bull. Amer. Math. Soc. 8 (1983), 337-339.

[4] A. S. Buch, Combinatorics of degeneracy loci, to appear.

[5] S. Fomin, S. Gelfand, and A. Postnikov, Quantum Schubert polynomials, J. Amer. Math. Soc. 10 (1997), 565-596.

[6] W. Fulton, Intersection Theory, Ergebnisse der Mathematik und ihrer Grenzgebiete (3), vol. 2, Springer-Verlag, Berlin, 1984, 1998.

[7] _ Flags, Schubert polynomials, degeneracy loci, and determinantal formulas, Duke Math. J. 65 (1992), 381-420.

[8] _ Universal Schubert polynomials, To appear in Duke Math. J., 1998.

[9] — Young Tableaux, London Mathematical Society Student Texts, vol. 35, Cambridge University Press, 1997.

[10] W. Fulton and P. Pragacz, Schubert varieties and degeneracy loci, to appear in Springer Lecture Notes, 1998.

[11] T. Józefiak, A. Lascoux, and P. Pragacz, Classes of determinantal varieties associated with symmetric and skew-symmetric matrices, Izv. Akad. Nauk SSSR Ser. Mat. 45 (1981), 662673.

[12] S. Katz and S. A. Strømme, "Schubert", a Maple package for intersection theory and enumerative geometry. Software and documentation available at ftp://ftp.math.okstate.edu/pub/schubert

[13] A. N. Kirillov and T. Maeno, Quantum double Schubert polynomials, quantum Schubert polynomials and Vafa-Intriligator formula, preprint, 1996.

[14] V. Lakshmibai and P. Magyar, Degeneracy schemes and Schubert varieties, preprint, 1997.

[15] A. Lascoux and M.-P. Schützenberger, Le monoïde plaxique, Noncommutative structures in algebra and geometric combinatorics (Naples, 1978) (Rome), Quad. "Ricerca Sci.", vol. 109, CNR, 1981, pp. 129-156.

[16] - Polynômes de Schubert, C. R. Acad. Sci. Paris Sér. I Math. 294 (1982), 447-450.

[17] I. G. Macdonald, Symmetric functions and Hall polynomials, Oxford University Press, 1979, 1995.

[18] P. Pragacz, Enumerative geometry of degeneracy loci, Ann. Sci. École Norm. Sup. (4) 21 (1988), 413-454.

Department of Mathematics, University of Chichgo, Chicago, IL 60637

E-mail address: abuch@math.uchicago.edu

Department of Mathematics, University of Chicago, Chicago, IL 60637

E-mail address: fulton@math.uchicago.edu 